\title{
HICSS-55 Minitrack Introduction Algorithm-Based Advisory in the Service Sector
}

\author{
Anke Schulz \\ LMU Munich \\ schulz@1mu.de
}

\author{
Anja Tuschke \\ LMU Munich \\ tuschke@bwl.lmu.de
}

\begin{abstract}
This minitrack focuses on the impact of algorithms on knowledge-intensive services - such as portfolio or legal advice. Designing and implementing algorithmbased technologies and business models in knowledgeintensive, risky contexts is complex and challenged by clients' considerable trepidations. Their successful design and implementation requires recognizing and meeting technical, organizational, as well as behavioral challenges. This minitrack sets out to explore such challenges facing algorithm-based advisory to better understand both, their foundations and solution approaches. It guides future research and practice by identifying real-world barriers to and enablers of algorithm-based innovation.
\end{abstract}

\section{Introduction}

Algorithm-based services fundamentally change how service providers operate and deliver value to clients. They offer many advantages to firms and their stakeholders in knowledge-intensive, risky contexts. However, their implementation faces considerable challenges ranging from process design to client reactions. Our minitrack called for different perspectives on the drivers of and solutions to these challenges. Our submitting authors responded with a variety of approaches, adressing different AI-assisted design options and stakeholders within the financial industry.

For HICSS-55 in 2022, our minitrack accepted one of several submissions. We were looking for highquality conference papers combining theoretical, methodological, and empirical rigor to advance research on algorithm-based advisory in the service sector. Our accepted paper uses regression analysis of a rich realworld data set as well as detailed expert interviews to investigate how private clients react to the introduction of robo advisory at a large German bank.

\section{Accepted Paper}

“Helping or Hindering? How Clients' Experiences Relate to Their Robo Advisory Use" is co-authored by Anke Schulz and Anja Tuschke from LMU Munich School of Management together with Alexander Ilgen from Deutsche Bank AG. The paper represents a unique collaboration combining insights from research and practice on robo advisory. Its analyses build on the theoretical foundations of knowledge and routines, seven in-depth interviews with bank advisors, as well as a recent data set on 11,302 clients from a leading German robo advisory provider. In their paper, the authors demonstrate how clients' reactions to robo advisory vary with their personal and business experiences at the focal bank. They find that knowledge and routines stemming from experience can both, align and misalign with robo advisory use, with particularly knowledge opening clients towards robo advisory use. The authors' analysis of a large, real-world data set allows them to provide complementary insights on the behavioral drivers behind use of algorithm-based services to those found in less risky contexts and study designs, e.g. experiments. For practitioners, the study provides data-backed results on which clients find robo advisory attractive, that can help bank advisors improve their recommendations.

\section{Outlook}

This minitrack focuses on how algorithms are transforming the service sector. We look forward to an interesting, scientifically sound, and practice-oriented discussion on the topic. We would like to thank all the authors for their submissions this year. 